\title{
Validação do School Liking and Avoidance Questionnaire para a população Portuguesa
}

\author{
Ana Raquel Ribeiro ${ }^{1}$, Ana Isabel Pereira ${ }^{1}$, \& Marta Pedro ${ }^{1}$ \\ ${ }^{1}$ Faculdade de Psicologia, Universidade de Lisboa
}

\begin{abstract}
Resumo: Este artigo apresenta o estudo de validação das versões portuguesas da Escala de Gosto pela Escola e Evitamento Escolar (SLAQ) para a crianças e para pais. Esta escala pretende avaliar o grau em que a criança gosta e evita a escola, na perspetiva da mesma e dos pais. A amostra é constituída por 394 alunos, do $2^{\circ}$ ao $4^{\circ}$ ano de escolaridade e respetivos pais. Na versão para crianças, a análise fatorial confirmatória revela uma estrutura bifatorial (Gosto pela Escola e Evitamento Escolar) equivalente à versão original e uma elevada consistência interna. Na versão para pais, não se verificou um ajustamento adequado entre os dados e a estrutura bifatorial original. Da análise fatorial exploratória, surgiu um ajustamento mais adequado de um modelo tri-fatorial da versão para pais (Gosto pela Escola; Evitamento Escolar; Comunicação sobre a Escola), com níveis bons de consistência interna.
\end{abstract}

\section{Palavras-chave: SLAQ; envolvimento afetivo escolar; crianças em idade escolar; estudos psicométricos.}

Validation of the School Liking and Avoidance Questionnaire for the Portuguese population: This study aimed to validate the Portuguese version of the School Liking and Avoidance Questionnaire (SLAQ) for children and parents. This scale pretends to evaluate the degree to which the child likes school and avoids school from the perspective of the child and parents. The sample consists of 394 students, from the 2nd to the 4th grade, and their parents. In the children's version, confirmatory factor analysis reveals an equivalent structure to the original two-factor version (School Liking and School Avoidance) and a high internal consistency. In the parent version, there was no adequate fit between the data and the original two-factor structure. From the exploratory factor analysis, a more appropriate adjustment of a three-factor model of the parent version (School Liking; School Avoidance; Communication about School) emerged, with good levels of internal consistency.

\section{Keywords: SLAQ; school affective involvement; school children; psychometric studies.}

A literatura sugere que os sentimentos que a criança desenvolve relativamente à escola nos primeiros anos têm tendência para persistirem no tempo (Ladd et al., 2000). Compreender como é construída esta ligação nos primeiros anos de escolaridade torna-se, assim, fundamental, pois além de ser uma fase de difícil adaptação é também um momento-chave para o desenvolvimento da motivação escolar e da perceção que a criança tem de si a nível académico (Hirvonen et al., 2016; Lynch \& Cicchetti, 1997).

0 presente artigo tem como objetivo a validação da Escala de Gosto pela Escola e de Evitamento Escolar (School Liking and Avoidance Questionnaire, Ladd \& Price, 1987). Esta é um instrumento que pretende avaliar o gosto pela escola, isto é, o grau em que a criança revela sentimentos positivos pela escola e o evitamento escolar, ou seja, a medida em que a criança demonstra o desejo de evitar a escola (Ladd et al., 1996). Para Ladd et al. (2000) é importante captar ambas as dimensões, uma vez que a ligação afetiva à escola é mais bem avaliada quando se consideram ambas as visões, positiva (gosto pela escola) e negativa (evitamento da escola). Assim sendo, níveis mais elevados de gosto pela escola significam que a criança detém sentimentos positivos sobre a mesma, enquanto que níveis mais elevados de evitamento escolar significam que a criança possui sentimentos negativos em relação à escola (Buhs \& Ladd, 2001).

A literatura identifica quatro tipos de envolvimento escolar, nomeadamente: comportamental, cognitivo, agenciativo e afetivo (Finn \& Zimmer, 2012; Fredricks et al., 2004; Reeve, 2013; Veiga et al., 2012), sendo que o gosto e o evitamento escolar se enquadram neste último tipo. Apesar de distintas, estas quatro dimensões encontram-se relacionadas. Um estudo de Ladd et al. (2000) com crianças em idade préescolar, observou uma correlação positiva entre atitudes e sentimentos positivos em relação à escola e indicadores precoces de envolvimento comportamental escolar. Um outro estudo com adolescentes, que analisou as inter-relações entre as dimensões comportamental, emocional e cognitiva do envolvimento

\footnotetext{
${ }^{1}$ Morada para correspondência: Ana Raquel Ribeiro, Faculdade de Psicologia, Universidade de Lisboa, Alameda da Universidade, Lisboa, Portugal 1649-013. E-mail: anaraquel_ribeiro@sapo.pt
} 
escolar, encontrou uma relação bidirecional entre as dimensões comportamental e emocional (Li \& Lerner, 2013).

O envolvimento comportamental e cognitivo com a escola e o seu impacto têm sido alvo de mais estudos (Ladd \& Dinella, 2009), por comparação à ligação afetiva da criança à escola que se encontra menos investigada. Para esta maior escassez de estudos tem contribuído a diversidade de construtos que descrevem a ligação afetiva da criança à escola na literatura (school belonging, school attachment, school liking, school bonding, school emotional engagement), a qual dificulta a interpretação e generalização de resultados empíricos (Ribeiro et al., 2019).

Segundo Ladd e colaboradores (2000), o gosto pela escola pode ser considerado um fator decisivo para a participação da criança em contexto de sala de aula. Ainda de acordo com os mesmos autores, também o sucesso académico precoce decorre de processos afetivos como o gosto pela escola. Os sentimentos que as crianças nutrem pela escola têm um grande impacto no seu sucesso escolar, pois sentimentos positivos aumentam a autoconfiança e o entusiamo relativamente à escola e a participação em atividades escolares (Valeski \& Stipek 2001). O gosto pela escola é, assim, considerado um aspeto fulcral uma vez que esta atitude positiva em relação à escola contribui para o desenvolvimento de uma aprendizagem autónoma (Ireson \& Hallam, 2005, Zhang et al., 2016).

De acordo com diversos estudos, a ligação afetiva da criança à escola encontra-se positivamente relacionada com o ajustamento escolar, bem como com a aprendizagem, sucesso, comportamento e motivação escolares, encontrando-se ainda negativamente relacionada com o absentismo (Abbott et al., 1998; Catalano et al., 2004; Eccles, 2009; Linnenbrink-Garcia \& Pekrun, 2011; Neel, \& Fuligni, 2013; Putwain et al., 2020; Reddy et al., 2003). Neste contexto, a promoção da ligação da criança à escola poderá constituir uma estratégia importante para a promoção da aprendizagem e aumento do sucesso escolar (Ladd, \& Dinella, 2009).

Segundo Fredricks et al. (2004), o envolvimento da criança na escola pode ter o seu início no sentimento de gostar da escola ou participar nas aulas e atividades escolares, a partir do qual poderá surgir na criança o compromisso ou investimento na escola, que, por sua vez, contribuirá para a diminuição da apatia e aumento da aprendizagem. Ladd, et al. (2000) referem que as primeiras manifestações dos sentimentos da criança relativamente à escola podem verificar-se na recetividade que a mesma demonstra em relação à escola, por exemplo, em comportamentos que demonstram vontade de se aproximar ou evitar a escola. Resultados de estudos com crianças em idade pré-escolar, revelam que atitudes e sentimentos positivos precoces em relação à escola (ex.: gostar da escola) correlacionam-se positivamente com a participação em sala de aula e o progresso académico (Ladd et al., 2000; Ladd, \& Dinella, 2009).

Num estudo longitudinal, que acompanhou crianças desde o 1. ao 8.. ano de escolaridade, com idades entre os 5 e os 13 anos, sobre os efeitos do envolvimento afetivo e comportamental precoce da criança à escola no sucesso escolar, os resultados indicam que a combinação de um alto envolvimento comportamental e afetivo ao longo dos primeiros anos escolares conduz a um maior progresso académico, contrariamente a um baixo envolvimento comportamental e afetivo que contribui negativamente para a aprendizagem e sucesso escolar (Ladd \& Dinella, 2009).

\section{Escala de Gosto pela Escola e de Evitamento Escolar}

Face às evidências que apontam para um impacto positivo da ligação afetiva da criança à escola na aprendizagem, adaptação à escola e sucesso escolar, optámos por validar para a população portuguesa a escala School Liking and Avoidance Questionnaire, de Ladd e Price (1987). 0 estudo e a validação de instrumentos que avaliam o envolvimento afetivo dos alunos com a escola no início da escolaridade vão de encontro aos objetivos do Programa Nacional de Promoção do Sucesso Educativo, que pretende fomentar um conjunto de práticas que possibilitem a antecipação e a prevenção do insucesso escolar, recorrendo à intervenção precoce. Este trabalho está ainda alinhado com as prioridades estratégicas da Agenda 2030 para o Desenvolvimento Sustentável, e em particular com o objetivo da Educação de Qualidade (ODS4), que pretende eliminar as altas taxas de retenção e o abandono escolar precoce, salientando a importância de se avaliar em níveis escolares iniciais a ligação afetiva da criança à escola (Ministério dos Negócios Estrangeiros, 2017). Também o Perfil do Aluno à Saída da Escolaridade Obrigatória (Ministério da Educação/ Direção Geral da Educação, 2017), documento que se constitui como referência e matriz para todas as escolas e o qual caracteriza o que se pretende que o aluno alcance no final do seu percurso escolar obrigatório em Portugal, vem realçar, igualmente, a importância do desenvolvimento pessoal do aluno o qual inclui o desenvolvimento da autoconfiança e motivação pela aprendizagem, essenciais para a evolução da autonomia, os quais dependem do envolvimento afetivo dos alunos com a escola.

Na versão original, o School Liking and Avoidance Questionnaire, é composto por duas subescalas, as quais avaliam a dimensão gosto pela escola e o evitamento escolar, existindo uma versão para crianças, para pais e para professores. A escala foi desenvolvida para a população norte-americana em idade pré- 
escolar e escolar, no entanto as suas propriedades têm sido avaliadas maioritariamente com crianças em idade pré-escolar e adolescentes. 0 estudo psicométrico da versão para crianças do SLAQ (Ladd \& Price, 1987) realizado com crianças em idade pré-escolar revelou uma consistência interna adequada ( $\alpha=.87$ e .91 , subescala gosto pela escola; .76 e .81, subescala evitamento escolar). Um estudo posterior desta escala com a população Norte-Americana que envolveu crianças do pré-escolar ao 6. $\stackrel{0}{ }$ ano de escolaridade (Smith, 2011) vai também no mesmo sentido, sugerindo níveis adequados de consistência interna para a versão crianças do SLAQ ( $\alpha=.88$ e 90, subescala gosto pela escola; .79 e .84, subescala evitamento escolar).

O SLAQ foi também estudado em cinco outros países (Grécia, Itália, Turquia, China e Japão) com amostras de crianças em idade pré-escolar (Nur \& Arnas, 2019; Tomada et al., 2005), em idade escolar (Vassilopoulos et al., 2018) e adolescentes (Honma \& Uchiyama, 2014; Zhang \& Eggum-Wilkens, 2018). Os estudos nestes países revelaram níveis adequados de consistência interna da subescala gosto pela escola ( $\alpha$ entre .79 e .92) e níveis mais variáveis em relação à escala de evitamento escolar ( $\alpha$ entre .68 e .87), bem como uma adequada validade convergente sustentada em correlações com variáveis académicas e sociais (Zhang \& Eggum-Wilkens, 2018).

A estrutura fatorial da versão para crianças foi avaliada em dois destes estudos realizados com crianças em idade pré-escolar, através de análises fatoriais exploratórias e confirmatórias. Os resultados indicaram uma estrutura de dois fatores consistente com a proposta da escala original (Nur \& Arnas, 2019; Tomada et al., 2005). Nenhum destes estudos explorou a estrutura fatorial da versão para pais do SLAQ.

Relativamente à versão para pais do SLAQ, um estudo realizado com crianças norte-americanas em idade escolar explorou a sua estrutura fatorial, através de uma análise fatorial confirmatória (Ladd \& Dinella, 2009). Os resultados deste estudo revelaram uma estrutura de um único fator com valores de ajustamento adequados e uma boa consistência interna $\alpha=.82$ e .95, primeiro e terceiro ano, respetivamente), calculada através da média da totalidade das respostas dos pais. Posteriormente, no estudo de Smith (2011), foi aplicada uma versão para pais do SLAQ com dois fatores (gosto pela escola e evitamento escolar), o qual revelou qualidades psicométricas adequadas ( $\alpha$ entre .76 e .81).

Assim, o presente estudo tem como objetivo analisar as características psicométricas da escala do gosto pela escola e evitamento escolar (versão para crianças e para pais) numa amostra de crianças e pais portugueses, recorrendo à análise fatorial, análise de correlações entre subescalas, análise de correlação entre as versões da escala para crianças e pais e, finalmente, à análise da consistência interna.

Tendo em consideração a versão original do SLAQ, colocam-se as seguintes hipóteses:

Hipótese 1: A versão portuguesa para crianças do SLAQ irá replicar a estrutura fatorial de duas dimensões do instrumento original;

Hipótese 2: Em ambas as versões (crianças e pais), a escala do gosto pela escola irá correlacionar-se negativamente com a escala de evitamento escolar.

Sendo que os resultados de estudos anteriores, os quais exploraram a estrutura fatorial da versão para pais do SLAQ, revelaram fraca consistência da mesma, considerou-se não se colocar nenhuma hipótese acerca da sua estrutura fatorial.

\section{MÉTODO}

\section{Participantes}

A amostra foi constituída por 394 alunos, com uma proporção equilibrada entre sexos (52.5\% raparigas). Os alunos tinham idades compreendidas entre 6 e $12(M=8.17, D P=1.06)$ e frequentavam a escola em diferentes graus de escolaridade (2. ano - 32.7\%, 3. ano - 34.8\% e 4. o ano - 32.5\%). Para além das crianças, participaram também 353 progenitores, na sua maioria mães (pais - 9.6\%, mães - 72.1\% e ambos - 7.9\%). Os participantes foram incluídos no estudo através do método de amostragem por conveniência, tendo sido convidados a participar todos os alunos entre o $2 .^{\circ}$ e o $4 .^{\circ}$ ano de escolaridade, no ano letivo 2018/19.

\section{Procedimento}

O SLAQ (versão para crianças e versão para pais) foi traduzido inicialmente por uma tradutora especializada em língua inglesa, sendo esta primeira tradução posteriormente revista por um segundo tradutor que sugeriu algumas alterações. De seguida, procedeu-se à retroversão da versão portuguesa por uma tradutora bilingue e, finalmente, os instrumentos foram aplicados a um pequeno grupo de crianças e pais para testar a clareza do vocabulário utilizado nos itens que compõem as escalas.

Posteriormente aos procedimentos anteriores, foi realizado o pedido de aprovação à Comissão Nacional de Proteção de Dados (CNPD) e à Direção Geral de Educação (Monitorização de Inquéritos em Meio Escolar). Após deferimento das entidades referidas, foi pedida colaboração às direções das escolas, professores de $1 .^{\circ}$ ciclo e pais. Os consentimentos informados foram entregues aos pais através dos filhos. 
Nestes consentimentos eram explicados os objetivos do estudo, os critérios de inclusão (frequentar a escola entre o 2. e o 4. ano de escolaridade) e a natureza voluntária do mesmo. Do total de 543 pais contactados, 394 aceitaram colaborar.

Aos alunos autorizados a participar, foi-lhes explicado o estudo pessoalmente e assegurada a confidencialidade dos dados. 0 protocolo de avaliação para crianças foi aplicado em contexto de sala de aula, na presença do professor, no final do 1. ${ }^{\mathrm{o}}$ período letivo. Uma das investigadoras fez a leitura do mesmo para cada grupo-turma. As crianças que foram identificadas pelo professor com necessidades educativas especiais que comprometiam a compreensão dos instrumentos foram excluídas a posteriori, apesar de terem participado no momento da aplicação do questionário. 0 protocolo de avaliação para pais foi enviado para casa, o qual depois de preenchido, foi devolvido em envelope fechado ao professor. No total foram devolvidos 38 questionários respondidos pelos pais, 284 pelas mães e 31 por ambos os progenitores.

\section{Instrumentos}

Escala de Gosto pela Escola e de Evitamento Escolar - versão para crianças. O SLAQ (Ladd \& Price, 1987), versão para crianças, consiste num instrumento de autorrelato que avalia como a criança se sente em relação à escola. A versão original da escala é constituída por 14 itens, divididos em duas subescalas: escala de gosto pela escola (9 itens) e escala de evitamento escolar (5 itens). No preenchimento do questionário, a criança tem de escolher, para cada item, uma de três respostas possíveis ("Sim" = 3, "Às vezes" = 2, "Não" = 1). Na escala de gosto pela escola encontram-se itens como "És feliz quando estás na escola?", "Gostas de ir para a escola?" ou "Quando acordas de manhã, sentes-te feliz por ires para a escola?". Os itens 2, 6 e 12 são pontuados de forma invertida. Na escala de evitamento escolar, incluem-se itens como "Gostavas de não ter que ir para a escola?" ou "Gostarias de ficar em casa em vez de ires para a escola?". Os resultados para cada escala são obtidos através da média da pontuação dos itens que compõem cada escala. Um resultado mais elevado na escala de gosto pela escola indica que a criança se sente ligada à mesma e, um resultado elevado na escala de evitamento escolar, indica que a criança evita/rejeita a mesma.

Escala de Gosto pela Escola e de Evitamento Escolar - versão para pais. A versão para pais do SLAQ consiste num instrumento de hetero-relato que avalia a perceção dos pais sobre como o seu filho se sente e se comporta, normalmente, em relação à escola. Este instrumento é constituído por 10 itens e, tal como na versão para crianças, tem duas escalas: escala de gosto pela escola (5 itens) e escala de evitamento escolar (5 itens). As escalas são cotadas numa escala de Likert de 5 pontos, variando de (1) Quase nunca a (5) Quase sempre. A escala de gosto pela escola inclui itens como "Deseja ir para a escola" ou "Fala-me sobre as coisas boas que aconteceram na escola". A escala de evitamento escolar inclui itens como "Inventa razões para ficar em casa e não ir à escola" ou "Parece ter medo de ir à escola".

Sucesso Escolar. 0 nível de sucesso escolar das crianças foi obtido através das notas de avaliação do primeiro período, referentes às três principais disciplinas (Português, Matemática e Estudo do Meio). Sendo que a avaliação é de caráter qualitativo, a mesma foi convertida numa uma escala de 4 pontos (1=Insuficiente a 4=Muito Bom) e, posteriormente, foi calculada a média das notas dos alunos.

\section{Análises estatísticas}

As análises estatísticas foram efetuadas com recurso ao software estatístico SPSS Statistics 25 e AMOS 24. Primeiramente, foi realizada uma análise descritiva dos dados obtidos (médias e desvios-padrão). Posteriormente, as análises estatísticas foram realizadas em cinco passos. Inicialmente, analisou-se a estrutura fatorial das versões portuguesas do SLAQ (versão criança e pais), recorrendo-se à análise fatorial confirmatória (AFC) com recurso ao método da Máxima Verossimilhança (ML).

Em primeiro lugar, estimou-se o modelo original bifatorial com duas variáveis latentes: gosto pela escola e evitamento escolar (Figuras 1 e 3). Em segundo lugar, o modelo original foi comparado com um modelo alternativo unifactorial (Figuras 2 e 4). Os índices $x^{2} / \mathrm{DF}$, Comparative Fit Index (CFI), Root Mean Square Error of Approximation (RMSEA) e Akaike Information Criterion (AIC) foram utilizados para avaliar o ajustamento dos modelos. Foi ainda utilizado o Expected Cross-Validation Índex (ECVI) para avaliar a diferença entre os modelos concorrentes testados, sendo que o valor menor de ECVI indica um modelo mais ajustado.

Em terceiro lugar, foi calculada a Variância Extraída Média (VEM). Esta análise foi realizada manualmente, seguindo as orientações de Fornell e Larcker (1981). A validade discriminante foi avaliada através da comparação da VEM de cada fator com o quadrado da correlação de Pearson $\left(R^{2}\right)$ entre fatores, existindo validade discriminante se VEM é superior ao $R^{2}$ (Fornell e Larcker, 1981). 
A validade de construto foi também avaliada através de análises correlacionais entre as subescalas do SLAQ, entre as versões do SLAQ para crianças e pais e entre as subescalas do SLAQ e o rendimento escolar dos alunos.

Em quarto lugar, procedeu-se à melhoria do modelo com o melhor ajustamento, considerando os valores dos índices de modificação (IM). Para a versão pais, procedeu-se ainda a uma análise fatorial exploratória através da análise dos componentes principais, com rotação Varimax. Por último, analisou-se a fiabilidade do SLAQ através da Fiabilidade Compósita (FC) e do Alpha de Cronbach. A Fiabilidade Compósita foi calculada manualmente e considerou-se uma fiabilidade adequada se FC $\geq .70$.

\section{RESULTADOS}

Os resultados das diferentes análises realizadas ao SLAQ, versão crianças e versão pais, serão apresentados separadamente.

\section{Escala de Gosto pela Escola e de Evitamento Escolar - versão crianças}

Na Tabela 1 são indicadas as estatísticas descritivas da versão para crianças do SLAQ apresentando as médias e desvios-padrão dos 14 itens que compõem o instrumento.

Tabela1. Análise Descritiva dos Itens do SLAQ (versão crianças)

\begin{tabular}{llc}
\hline Itens & Média & Desvio-padrão \\
\hline Escala de Gosto pela Escola & & .47 \\
1. A escola é divertida? & 2.76 & .54 \\
2. A escola faz-te sentir com vontade de chorar?* & 2.76 & .65 \\
4. És feliz quando estás na escola? & 2.60 & .67 \\
6. Detestas a escola?* & 2.65 & .62 \\
7. Gostas de estar na escola? & 2.68 & .67 \\
8. Gostas de ir para a escola? & 2.62 & .66 \\
10. A escola é um sítio divertido para se estar? & 2.62 & .71 \\
11. Quando acordas (...) sentes-te feliz por ires para a escola? & .95 \\
12. A escola não presta?* & 2.46 & .81 \\
\hline Escala de Evitamento Escolar & 2.12 & .80 \\
3. Gostavas de não ter que ir para a escola? & & .81 \\
5. Gostarias que a tua mãe ou o teu pai te deixassem ficar em casa em & 1.61 & .81 \\
vez de ires para a escola? & 1.52 & .66 \\
9. Gostarias de ficar em casa em vez de ires para a escola? & 1.63 & 2.13 \\
13. Sentes-te mais feliz quando são horas de ir embora da escola (...)? & 1.33 & \\
14. Pedes (...) para ficares em casa em vez de ires para a escola? & & \\
\hline
\end{tabular}

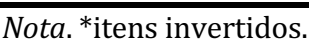

Os resultados da análise descritiva indicam valores médios dos itens da escala de gosto pela escola entre 2.12 (item 12 "A escola não presta?") e 2.76 (item 1 "A escola é divertida?" e item 2 "A escola faz-te sentir com vontade de chorar?"). Por seu lado, na escala de evitamento escolar observam-se valores médios entre 1.33 (item 14 "Pedes aos teus pais para ficares em casa em vez de ires para a escola?") e 2.13 (item 13 "Sentes-te mais feliz quando são horas de ir embora da escola e ir para casa?").

Para se analisar a estrutura fatorial da versão portuguesa do SLAQ, foi testada a estrutura bifatorial proposta pelos autores da escala original através de análise fatorial confirmatória. Na Figura 1 é apresentado o modelo de dois fatores, o qual pressupõe a existência de correlação entre si (gosto pela escola e evitamento escolar) e na Figura 2 encontra-se um modelo alternativo unifatorial, o qual foi comparado com o modelo original. 


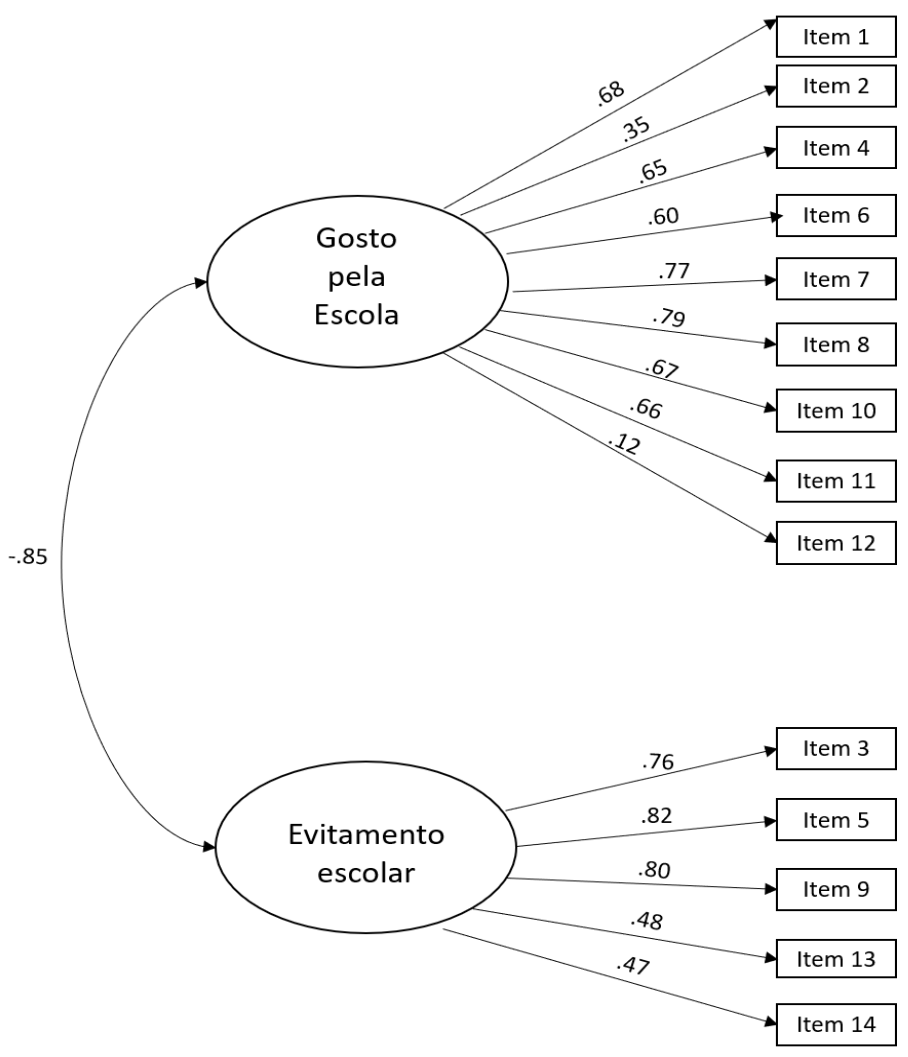

Figura 1. Modelo bifatorial da versão original - versão crianças.

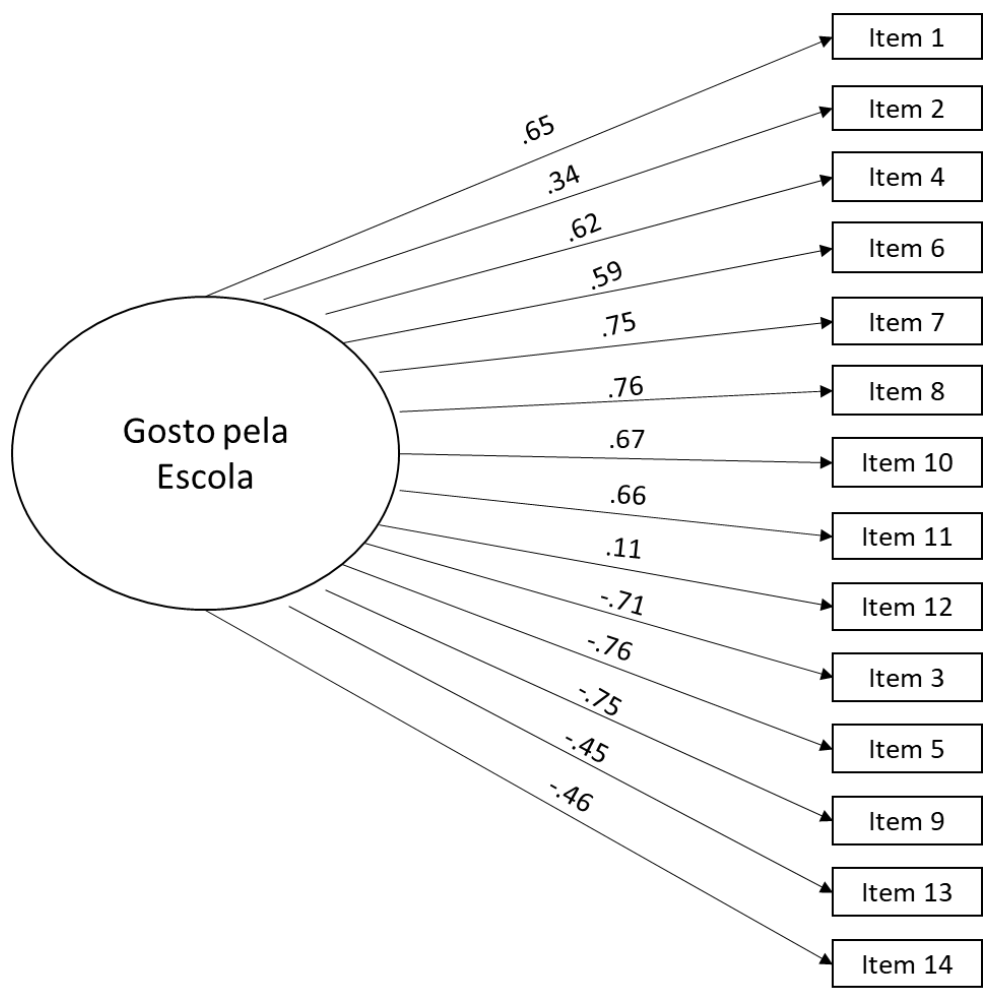

Figura 2. Modelo alternativo unifatorial.

Os índices que resultam da avaliação da qualidade do ajustamento dos dois modelos encontram-se na Tabela 2. 
Tabela 2. Índices da qualidade do ajustamento dos Modelos

\begin{tabular}{lccccccc}
\hline & $\mathrm{X}^{2}$ & DF & P & CFI & RMSEA & AIC & ECVI \\
\hline $\begin{array}{l}\text { Modelo } \\
\text { bifatorial }\end{array}$ & 191.09 & 76 & .000 & .95 & .06 & 277.09 & .71 \\
$\begin{array}{l}\text { Modelo } \\
\text { unifatorial }\end{array}$ & 281.16 & 77 & .000 & .90 & .08 & 337.16 & .86 \\
\hline
\end{tabular}

Alguns índices de qualidade de ajustamento do modelo bifatorial sugerem um ajustamento adequado (Comparative Fit Index,Root Mean Square Error of Approximation). 0 índice de ajustamento $x^{2} / \mathrm{DF}$ é considerado razoável, pois apresenta um valor de 2.51. Embora o modelo unifatorial apresente alguns indicadores que sugerem um nível adequado de ajustamento, os valores de $x^{2}$ e de ECVI são mais baixos.

Verifica-se que os resultados da Variância Extraída Média (VEM), calculados manualmente a partir dos pesos fatoriais dos itens, encontram-se abaixo do aceitável com valores inferiores a 0.5 (gosto pela escola $=0.39$; evitamento escolar $=0.47$ ) (Fornell \& Larcker, 1981). Quanto à validade discriminante, verifica-se que a VEM de cada fator é inferior ao $\mathrm{R}^{2}(.48)$ entre os fatores.

A validade de construto foi analisada a partir da correlação de Pearson entre o gosto pela escola e o evitamento escolar e entre estas duas variáveis e o rendimento escolar da criança. Os resultados indicam uma correlação negativa significativa de forte magnitude $(r=-.69, p<.01)$ entre o gosto pela escola e o evitamento escolar.

Os resultados indicam uma correlação positiva significativa de baixa magnitude $(r=.157, p<.01)$ entre o gosto pela escola e o sucesso escolar e uma correlação negativa significativa de baixa magnitude $(r$ $=-.126, p<.05$ ) entre o evitamento escolar e o sucesso escolar.

Na Tabela 3 encontram-se as estatísticas descritivas das duas escalas, bem como os valores da consistência interna. Os resultados obtidos indicam níveis elevados de gosto pela escola e níveis moderados-baixos de evitamento escolar. Quanto à fiabilidade, o alfa de Cronbach de cada subescala revelase adequado, sendo consistente com os resultados da análise da Fiabilidade Compósita (FC) (gosto pela escola = 0.84; evitamento escolar $=0.81$ ).

Tabela 3. Médias, desvios-padrão e consistência interna das subescalas

\begin{tabular}{llll}
\hline & Média & Desvio-padrão & Consistência Interna \\
\hline Gosto pela Escola & 2.56 & .42 & 0.80 \\
Evitamento Escolar & 1.64 & .58 & 0.79 \\
\hline
\end{tabular}

Escala de Gosto pela Escola e de Evitamento Escolar - versão pais

As estatísticas descritivas da SLAQ, versão para pais, encontram-se indicadas na Tabela 4.

Tabela 4. Análise descritiva dos itens do SLAQ (versão pais)

\begin{tabular}{lcc}
\hline Itens & Média & Desvio-padrão \\
\hline Escala de Gosto pela escola & & .72 \\
1. Gosta das atividades e eventos escolares. & 4.37 & .83 \\
2. Deseja ir para a escola. & 4.39 & .91 \\
3. Fala da escola de uma forma negativa.* & 4.33 & .84 \\
4. Fala-me sobre as coisas boas (...) na escola. & 4.19 & .79 \\
5. Fala-me de situações engraçadas da escola.** & 4.27 & .64 \\
\hline Escala de Evitamento Escolar & & .56 \\
1. Inventa razões para não ir à escola.** & & .64 \\
2. Parece ter medo de ir à escola. & 1.28 & .63 \\
3. Fica perturbado quando vai para a escola. ${ }^{* *}$ & 1.22 & .77 \\
4. Pede para ficar em casa e não ir à escola. & 1.28 & 1.29 \\
5. Queixa-se por ter que ir para a escola. & 1.40 & \\
\hline
\end{tabular}

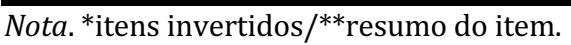


$\mathrm{Na}$ escala de gosto pela escola, os resultados indicam valores médios entre 4.19 (item 4 "Fala-me sobre as coisas boas que aconteceram na escola.") e 4.39 (item 2 "Deseja ir para a escola."). Relativamente à escala de evitamento escolar, a média mínima é de 1.22 (item 2 "Parece ter medo de ir para a escola.") e a média máxima de 1.40 (item 5 “Queixa-se por ter que ir para a escola.").

Na Figura 3 é apresentado o modelo de estrutura bifatorial apresentada pelos autores da escala, o qual pressupõe igualmente a existência de correlação entre os dois fatores (gosto pela escola e evitamento escolar) e, na Figura 4, é apresentado um modelo unifatorial para comparação.

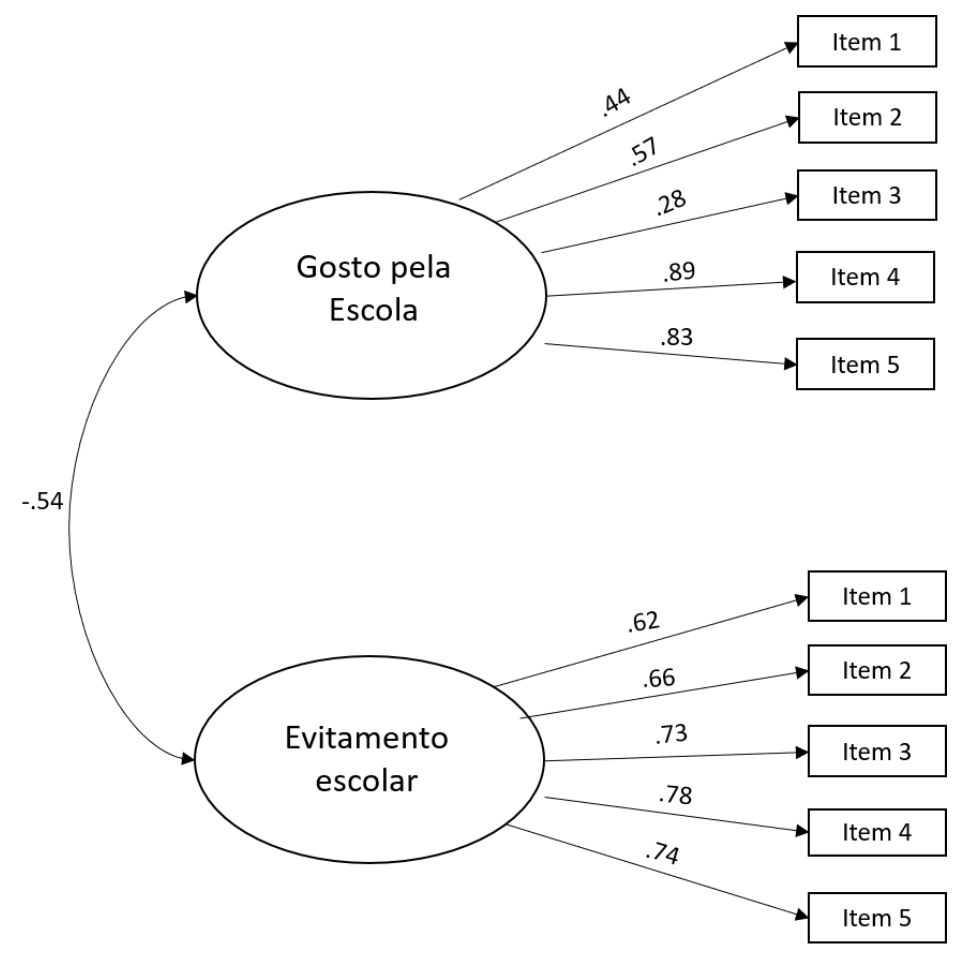

Figura 3. Modelo bifatorial da versão original - versão pais.

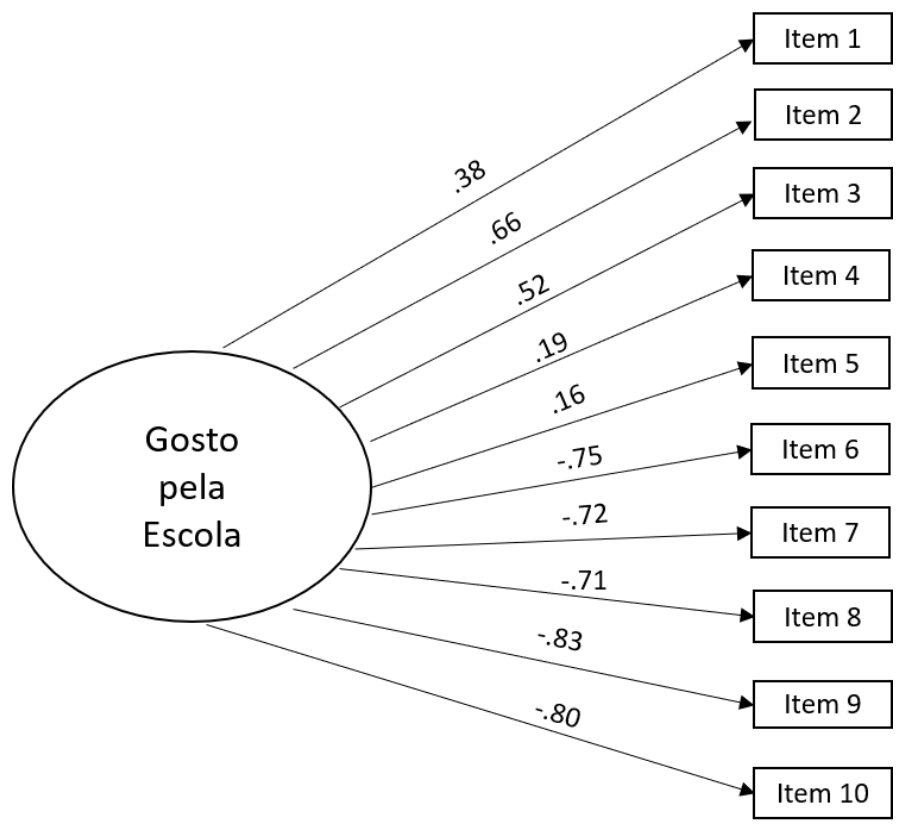

Figura 4. Modelo alternativo unifatorial. 
Os resultados da análise fatorial confirmatória do modelo bifatorial original indicam a falta de ajustamento entre os dados e a estrutura do modelo apresentado $\left(x^{2} / \mathrm{DF}=7.25, \mathrm{CFI}=0.48, \mathrm{RMSEA}=0.13\right)$, situando-se no nível inaceitável. Seguidamente, fez-se a comparação entre o modelo bifatorial e o modelo unifatorial ao nível dos índices de ajustamento.

Os índices que resultam da avaliação da qualidade do ajustamento do modelo unifatorial, determinado através do método da estimativa da máxima verosimilhança, demonstram um ajustamento inadequado com $x^{2}$ /DF igual a 15.08, um CFI igual a 0.72 e um RMSEA igual a 0.19, situando-se no nível inaceitável.

Como não se observou um bom ajustamento de dados do modelo original bifatorial, foi necessário reespecificá-lo com o objetivo de se conseguir uma melhoria significativa no seu ajustamento e validar um modelo teórico. Procedeu-se à melhoria do modelo correlacionando os erros/resíduos dos itens mais significativos (IM > 11), tendo como base os índices de modificação (IM) calculados pelo AMOS. As correlações mais significativas verificaram-se entre os erros dos itens 8 e 9 (IM = 30) e dos itens 7 e 10 (IM =21).

Os índices de qualidade de ajustamento resultantes da análise fatorial confirmatória do modelo melhorado dos dois fatores não apresentam, igualmente, valores de ajustamento adequados $\left(X^{2} / \mathrm{DF}=5.6\right.$; CFI = 0.67 e RMSEA = 0.11) constatando-se, assim, uma inadequação da versão dos pais.

Sendo que os resultados não apoiam a validade fatorial do SLAQ (versão pais), procedeu-se à análise fatorial exploratória do mesmo. A adequação dos dados recolhidos através do instrumento em estudo para a realização de uma análise fatorial exploratória foi avaliada através do coeficiente de Kaiser-Meyer-Olkin (KMO) e do Teste de Esfericidade de Bartlett. Os resultados do coeficiente KMO (= 0.82) e do Teste de Esfericidade de Bartlett $\left(\chi^{2}=1797.66 ; p<.0001\right)$ confirmaram que os dados recolhidos são adequados para a análise fatorial exploratória. Para a realização da mesma, utilizou-se o método de extração de análise de Componente Principais, sem decisão prévia de componentes a reter e, verificou-se, recorrendo ao critério de Kaiser-Guttman, a identificação de dois fatores com valores próprios superiores a um. Contudo, através da análise do gráfico de escarpa e dos pontos de inflexão sugeridos no mesmo, selecionaram-se três fatores. Posteriormente, foi feita a análise fatorial exploratória recorrendo ao método de extração de Componente Principal e rotação Varimax para a extração

de três fatores. Para definir a inclusão de um item num fator, considerou-se o critério do peso fatorial ser superior a 0.3. Na Tabela 5 encontram-se descritos os pesos fatoriais dos itens que compõem cada um dos fatores. 0 Fator 1 foi denominado Evitamento Escolar, o Fator 2 foi intitulado Comunicação sobre a Escola e, o Fator 3, Gosto pela Escola.

Tabela 5. Cargas fatoriais dos itens do SLAQ (versão pais)

\begin{tabular}{|c|c|c|c|c|}
\hline & Item & 10 Fator & 2o Fator & 3 Fator \\
\hline \multirow{11}{*}{$\underset{\varpi}{\stackrel{一}{\leftrightarrows}}$} & Pede para ficar em casa e não ir à escola. & 0.83 & & \\
\hline & Queixa-se por ter que ir para a escola. & 0.82 & & \\
\hline & Parece ter medo de ir à escola. & 0.80 & & \\
\hline & Fica perturbado quando vai para a escola. & 0.78 & & \\
\hline & Inventa razões para não ir à escola. & 0.73 & & \\
\hline & Fala da escola de uma forma negativa. & -0.56 & & \\
\hline & Fala-me de situações engraçadas da escola. & & 0.93 & \\
\hline & Fala-me sobre as coisas boas (...) na escola. & & 0.92 & \\
\hline & Gosta das atividades e eventos escolares. & & & 0.89 \\
\hline & Deseja ir para a escola. & & & 0.65 \\
\hline & Variância Expl. \% & 43.51 & 17.59 & 8.73 \\
\hline
\end{tabular}

Após a análise exploratória, a qual revelou uma estrutura de 3 fatores, realizou-se uma análise confirmatória do modelo tri-fatorial através do AMOS24.

Os resultados obtidos numa primeira análise fatorial confirmatória do modelo de três fatores indicaram a não existência de um bom ajustamento do mesmo ( $x^{2} / \mathrm{DF}=4.05$; CFI $=.76$ e RMSEA $=.09$ ), sendo necessário reespecificá-lo com o objetivo de se conseguir uma melhoria significativa no seu ajustamento e validar um modelo teórico. A melhoria do modelo correlacionando os erros/resíduos dos itens mais significativos (IM > 11), teve como base os índices de modificação (IM) calculados pelo AMOS. As correlações mais significativas verificaram-se entre os erros dos itens 8 e 9 (IM=28) e dos itens 7 e 10 $(\mathrm{IM}=23)$. 
Os resultados da AFC do modelo melhorado apresentam melhorias significativas nos índices de qualidade de ajustamento. Embora o valor de $x^{2} / \mathrm{DF}$ (2.33) não seja aceitável, os valores do CFI (Comparative Fit Index $=0.910$ ) e o RMSEA (Root Mean Square Error of Approximation $=0.06$ ) são considerados bons. Os resultados demonstram um ajustamento mais adequado do modelo tri-fatorial do SLAQ (versão pais) para a população portuguesa.

Os resultados da Variância Extraída Média (VEM), calculados manualmente a partir dos pesos fatoriais dos itens, apresentam uma validade de estrutura interna adequada com valores superiores a 0.5 (evitamento escolar = 0.54; comunicação sobre a escola $=0.78$; gosto pela escola $=0.56$ ) (Fornell \& Larcker, 1981). No que diz respeito à validade discriminante, os três fatores apresentam valores de VEM superiores ao $\mathrm{R}^{2}$ entre os mesmos (evitamento escolar e comunicação sobre a escola $=.02$, evitamento escolar e gosto pela escola $=.23$ e comunicação sobre a escola e gosto pela escola $=.07$ ).

A validade de construto foi também estudada a partir da análise das correlações entre as três subescalas através do coeficiente de Pearson, verificando-se associações estatisticamente significativas. Existe uma correlação negativa de fraca magnitude entre a subescala evitamento escolar e a subescala comunicação sobre a escola $(r=-.13, p<.05)$ e uma correlação negativa moderada entre a subescala evitamento escolar e a subescala gosto pela escola $(r=-.48, p<.01)$. Entre a subescala comunicação sobre a escola e a subescala gosto pela escola observa-se a existência de uma correlação positiva de fraca magnitude $(r=.27, p<.01)$.

Foi analisada a associação entre os três fatores da versão para pais e o rendimento escolar da criança através do coeficiente de correlação de Pearson. Os resultados indicam que existe uma correlação negativa significativa de baixa magnitude entre o evitamento escolar e o sucesso escolar $(r=-.26, p<.01)$ e uma correlação positiva significativa fraca entre o gosto pela escola e o sucesso escolar $(r=.30, p<.01)$. A associação entre a comunicação sobre a escola e o sucesso escolar $(r=.09, p>.01)$ não é significativa.

Na análise da relação entre as subescalas do SLAQ (Tabela 6) da versão para crianças e versão para pais, verificam-se correlações estatisticamente significativas entre algumas subescalas. A subescala comunicação sobre a escola não apresenta uma correlação estatisticamente significativa com nenhuma subescala da versão para crianças. Verificam-se correlações positivas de magnitude moderada entre a subescala gosto pela escola (versão pais) e correlações negativas de magnitude moderada as subescalas gosto pela escola (versão crianças) e evitamento escolar (versão crianças). A subescala evitamento escolar (versão para pais) apresenta uma correlação negativa de magnitude baixa com a subescala gosto pela escola da versão para crianças e uma correlação positiva de magnitude baixa com a subescala evitamento escolar da versão para crianças.

Tabela 6. Correlações entre as escalas da versão para pais e da versão para crianças

\begin{tabular}{lcccc}
\hline & & \multicolumn{3}{c}{ Versão Pais } \\
\cline { 3 - 4 } & & Evitamento escolar & $\begin{array}{c}\text { Comunicação sobre } \\
\text { escola }\end{array}$ & Gosto pela Escola \\
\hline Versão & Gosto pela Escola & $-.22^{* *}$ & -.04 & $.31^{* *}$ \\
Crianças & Evitamento escolar & $.19^{* *}$ & -.00 & $-.32^{* *}$ \\
\hline
\end{tabular}

** A correlação é significativa no nível 0.01 ( 2 extremidades).

Na Tabela 7 encontram-se as estatísticas descritivas das três componentes e os níveis de consistência interna. Verificam-se níveis mais elevados nas subescalas comunicação sobre a escola e gosto pela escola. Na subescala evitamento escolar, a média de pontuação de resposta é mais baixa, verificandose que os pais percecionam baixo evitamento escolar nos filhos.

Tabela 7. Médias, desvios-padrão e consistência interna das subescalas

\begin{tabular}{lccc}
\hline & Média & Desvio-padrão & Consistência Interna \\
\hline Evitamento Escolar & 1.8 & .41 & .85 \\
Comunicação sobre a escola & 4.23 & .79 & .86 \\
Gosto pela Escola & 4.38 & .69 & .64 \\
\hline
\end{tabular}

No que diz respeito à fiabilidade, observa-se uma Fiabilidade Compósita elevada nos três fatores (evitamento escolar $=0.87$; comunicação sobre a escola $=0.87$; gosto pela escola $=0.70$ ). 
O alfa de Cronbach da subescala evitamento escolar e o alfa de Cronbach da subescala comunicação sobre a escola são considerados bons. 0 alfa da subescala gosto pela escola, embora mais baixo, pode ser considerado aceitável tendo em conta o número baixo de itens.

\section{DISCUSSÃO}

Este estudo teve como objetivos o desenvolvimento e validação das versões portuguesas para crianças e para pais da Escala de Gosto pela Escola e Evitamento Escolar para a população portuguesa (Ladd et al., 2000; Ladd \& Price, 1987). Vários estudos evidenciam a importância dos sentimentos que a criança desenvolve pela escola e o impacto desses mesmos sentimentos na ligação afetiva à escola, sucesso educativo e comportamento (Catalano et al., 2004; Eccles, 2009; Reddy et al., 2003).

Segundo alguns autores, a ligação da criança à escola constitui-se como um fator positivo para a motivação escolar, aprendizagem, sucesso e ajustamento escolar (Abbott et al., 1998; Catalano et al., 2004; Eccles, 2009; Hirvonen et al., 2016; Lynch \& Cicchetti, 1997; Neel, \& Fuligni, 2013; Reddy et al., 2003). Perante estes dados, surgiu o interesse pela validação da Escala de Gosto pela Escola e Evitamento Escolar.

Relativamente à estrutura fatorial, na versão para crianças da Escala de Gosto Pela Escola e Evitamento Escolar, os resultados psicométricos obtidos revelaram-se muito semelhantes aos obtidos em estudos anteriores (Honma \& Uchiyama, 2014; Ladd \& Price, 1987; Smith, 2011; Zhang \& Eggum-Wilkens, 2018). Na versão para crianças, a análise fatorial confirmatória revelou a existência de uma estrutura de dois fatores distintos (gosto pela escola e evitamento escolar), resultados consistentes com a versão original e outros estudos de adaptação da escala (Honma \& Uchiyama, 2014; Ladd \& Price, 1987; Smith, 2011; Tomada et al., 2005; Zhang \& Eggum-Wilkens, 2018; Nur \& Arnas, 2019), bem como com a hipótese colocada pelas autoras. Os resultados sugerem algumas fragilidades da escala a nível da sua validade fatorial (pesos inferiores a 0.5 em alguns itens), da validade convergente e validade discriminante. Uma das explicações para a obtenção destes resultados poderá ser o facto desta escala incluir apenas três níveis de resposta. No futuro, uma hipótese a considerar será aumentar as alternativas da escala de resposta da versão para crianças.

$\mathrm{Na}$ versão para pais, os dados obtidos neste estudo não apoiaram a estrutura, originalmente proposta de dois fatores (Ladd et al., 2000; Smith, 2011), nem a estrutura unifatorial de Ladd e Dinella (2009). A análise fatorial exploratória identificou uma estrutura composta por três fatores distintos (evitamento escolar, comunicação sobre a escola e gosto pela escola), com um ajustamento mais adequado para a população portuguesa.

Os resultados da correlação de Pearson entre os fatores gosto pela escola e evitamento escolar para ambas as versões revelaram uma associação negativa, de magnitude moderada a elevada, apoiando uma adequada validade do construto da escala (Buhs \& Ladd, 2001; Ladd et al., 2000;).

Verificaram-se ainda associações estatisticamente significativas entre as duas versões do SLAQ e o sucesso escolar, embora na sua maioria de fraca magnitude. Estes resultados vão no sentido de outros estudos que evidenciam, igualmente, uma associação entre os sentimentos que as crianças nutrem pela escola e o sucesso escolar, em idade escolar (Ladd et al., 2000; Ladd \& Dinella, 2009).

$\mathrm{Na}$ análise da relação entre as subescalas da versão para crianças e para pais, os resultados mostraram relações significativas entre as subescalas gosto pela escola e evitamento escolar de ambas as versões (Smith, 2011). A ausência de associações estatisticamente significativas entre a subescala comunicação sobre a escola e as duas subescalas da versão para crianças é inesperada e deverá ser averiguada em estudos futuros.

No que diz respeito à fiabilidade dos instrumentos, quer na versão para crianças quer para pais, obtiveram-se níveis de consistência interna adequados, à semelhança de outros estudos (Nur \& Arnas, 2019; Ladd \& Dinella, 2009; Tomada et al., 2005; Vassilopoulos et al., 2018).

É essencial referir algumas limitações deste estudo. Apesar de a amostra apresentar uma dimensão considerável, apenas foram estudadas crianças do 1.ำ ciclo. Os primeiros anos escolares são cruciais para uma adaptação positiva à escola, no entanto, existem alterações significativas a nível escolar a partir do 5.ำ ano de escolaridade, as quais requerem novas adaptações que podem modificar o sentimento de ligação à escola. Alguns estudos observaram que os sentimentos em relação à escola vão-se tornando menos positivos ao longo do tempo à medida que as crianças desenvolvem expetativas mais realistas em relação à mesma (Ladd et al., 2017; Smith, 2011). Neste sentido, sugere-se a realização de estudos de validação deste instrumento para idades superiores a 10 anos ou ao quarto ano de escolaridade. Ainda relacionado com a amostra, outra limitação consiste no contexto sociocultural. Os dados foram recolhidos numa amostra concentrada no interior do país, a partir de agrupamentos de escolas de dimensão pequena. É importante realizar mais estudos utilizando uma amostra de crianças e pais de contextos socioculturais diversificados. 
Consideramos igualmente pertinente a realização de estudos que analisem a relação existente entre o gosto da criança pela escola/evitamento escolar e outras variáveis do funcionamento escolar e social da criança. Estudos indicam que um maior envolvimento afetivo contribui para um melhor ajustamento escolar (Catalano et al., 2004; Connell \& Wellborn, 1991; Fernandes et al., 2014; Skinner \& Belmont, 1993). Sintetizando, os resultados do presente estudo relativamente à versão portuguesa para crianças do SLAQ são consistentes com os estudo prévios conduzidos noutros países. Relativamente à versão para pais, propomos a estrutura tri-fatorial do SLAQ por se considerar o modelo estrutural mais adequado e viável para a população portuguesa, apesar de possuir uma estrutura fatorial pouco consistente e pouco coerente com análises anteriores, sugerindo-se mais estudos a este respeito. De um modo geral, devido a algumas limitações relativamente às suas características psicométricas, ambas as versões do SLAQ deverão ser usadas com cautela e como instrumento complementar na avaliação individual do aluno.

Sendo que não temos conhecimento até à data de instrumentos em Portugal que avaliem a ligação afetiva da criança à escola, este estudo vem contribuir para a diminuição dessa lacuna. A finalidade principal deste estudo é a aplicabilidade deste instrumento, no futuro, a crianças em idade escolar e respetivos pais. O SLAQ pode contribuir para a compreensão do processo de adaptação da criança à escola e para a prevenção de um mau ajustamento educacional e psicológico (Ladd et al., 1999). Como refere Şahin-Sak (2018), é fundamental estudar os sentimentos das crianças em relação à escola para que seja possível responder às necessidades escolares das crianças de forma eficiente e, também, construir e desenvolver programas escolares eficazes que atuem precocemente (ex.: transição do pré-escolar para o primeiro ciclo), para que as crianças desenvolvam um maior gosto pela escola e consequentemente, tenham maior sucesso académico e menos problemas de comportamento (Schneider et al., 2014).

\section{REFERÊNCIAS}

Abbott, R. D., O’Donnell, J., Hawkins, J. D., Hill, K. G., Kosterman, R., \& Catalano, R. F. (1998). Changing teaching practices to promote achievement and bonding to school. The American Journal of Orthopsychiatry, 68(4), 542-552. https://doi.org/10.1037/h0080363

Buhs, E. S., \& Ladd, G. W. (2001). Peer rejection as an antecedent of young children's school adjustment: An examination of mediating processes. Developmental Psychology, 37(4), 550-560. https://doi.org/10.1037/0012-1649.37.4.550

Catalano, R. F., Haggerty, K. P., Oesterle, S., Fleming, C. B., \& Hawkins, J. D. (2004). The importance of bonding to school for healthy development. Journal of School Health, 74(7), 252-261. https://doi.org/10.1111/j.1746-1561.2004.tb08281.x

Connell, J. P., \& Wellborn, J. G. (1991). Competence, autonomy and relatedness: A motivational analysis of self-system processes. In M. Gunnar \& L. A. Sroufe (Eds.), Minnesota Symposium on Child Psychology: Vol. 23. Self processes in development (pp. 43-77). University of Chicago Press.

Direção-Geral da Educação, M. da E. (2017). Perfil dos alunos à saída da escolaridade obrigatória. Editorial Do Ministério Da Educação e Ciência, 1-30. Retrieved from http://www.dge.mec.pt/sites/default/files/Curriculo/Projeto_Autonomia_e_Flexibilidade/perfil_d os_alunos.pdf

Eccles, J. S. (2009). Who am I and what am I going to do with my life? Personal and collective identities as $\begin{array}{lllll}\text { motivators of } \quad \text { Educational } & \text { Psychologist, } & \text { 44, } & \text { 789. }\end{array}$ https://doi.org/10.1080/00461520902832368

Fernandes, H. R., Caldeira, S. N., \& Veiga, F. H. (2014). Envolvimento do aluno na escola e comportamento disruptivo. Revista Ibero-americana de Educação, 66(1), 1-12. https://doi.org/10.35362/rie661293

Finn, J. D., \& Zimmer, K. S. (2012). Student engagement: What is it? Why does it matter? In Christenson, S. L., Reschly, A. L., Wylie, C. (Eds.), Handbook of research on student engagement (pp. 97-132). https://doi.org/10.1007/978-1-4614-2018-7_5

Fornell, C., \& Larcker, D. F. (1981). Evaluating structural equation models with unobservable variables and measurement error. XVi(February), 39-50. https://doi.org/10.2307/3151312

Fredricks, J. A., Blumenfeld, P. C., \& Paris, A. H. (2004). School engagement: Potential of the concept, state of the evidence. Review of Educational Research, 74(1), 59-109. https://doi.org/10.3102/00346543074001059

Hirvonen, R., Torppa, M., Nurmi, J. E., Eklund, K., \& Ahonen, T. (2016). Early temperament and age at school entry predict task avoidance in elementary school. Learning and Individual Differences, 47, 1-10. https://doi.org/10.1016/j.lindif.2015.12.012

Honma, Y., \& Uchiyama, I. (2014). Emotional engagement and school adjustment in late childhood: The relationship between school liking and school belonging in japan. Psychological Reports, 114(2), 496-508. https://doi.org/10.2466/21.10.pr0.114k19w7 
Ireson, J., \& Hallam, S. (2005). Pupils' liking for school: Ability grouping, self-concept and perceptions of teaching. British Journal of Educational Psychology, 75, 297-311. https://doi.org/10.1348/000709904X24762

Ladd, G. W., Birch, S. H., \& Buhs, E. S. (1999). Children's social and scholastic lives in kindergarten: Related spheres of influence? Child Development, 70(6), 1373-1400. https://doi.org/10.1111/14678624.00101

Ladd, Gary W., Buhs, E., \& Seid, M. (2000). Children's initial sentiments about kindergarten: Is school liking an antecedent of early childhood classroom participation and achievement. Merrill-Palmer Quarterly, 46(2), 255-279.

Ladd, G. W., \& Dinella, L. M. (2009). Continuity and change in early school engagement: Predictive of children's achievement trajectories from first to eighth grade? Journal of Educational Psychology, 101(1), 190-206. https://doi.org/10.1037/a0013153

Ladd, G. W., Ettekal, I., \& Kochenderfer-Ladd, B. (2017). Peer victimization trajectories from kindergarten through high school: Differential pathways for children's school engagement and achievement? Journal of Educational Psychology, 109(6), 826-841. https://doi.org/10.1037/edu0000177

Ladd, G. W., Kochenderfer, B. J., \& Coleman, C. C. (1996). Friendship quality as a predictor of young children's early school adjustment. Child Development, 67(3), 1103-1118. https://doi.org/10.1111/j.1467-8624.1996.tb01785.x

Ladd, G. W., \& Price, J. M. (1987). Predicting children's social and school adjustment following the transition from preschool to kindergarten. Child Development, 58(5), 1168. https://doi.org/10.2307/1130613

Li, Y., \& Lerner, R. M. (2013). Interrelations of behavioral, emotional, and cognitive school engagement in high school students. Journal of Youth and Adolescence, 42(1), 20-32. https://doi.org/10.1007/s10964-012-9857-5

Linnenbrink-Garcia, L., \& Pekrun, R. (2011). Students' emotions and academic engagement: Introduction to the special issue. Contemporary Educational Psychology, 36(1), 1-3. https://doi.org/10.1016/j.cedpsych.2010.11.004

Lynch, M. \& Cicchetti, D. (1997). Children's relationships with adults and peers: An examination of elementary and junior high school students. Journal of School Psychology 35(1), 8199https://doi.org/10.1016/S0022-4405(96)00031-3

Ministério dos Negócios Estrangeiros (2017). Relatório nacional sobre a implementação da Agenda 2030 $\begin{array}{lllll}\text { para } o & \text { Desenvolvimento } & \text { Sustentável. } & \text { 86-88. }\end{array}$ https://sustainabledevelopment.un.org/content/documents/15766Portugal2017_EN_REV_FINAL_ 29_06_2017.pdf

Neel, C. G. O., \& Fuligni, A. (2013). A longitudinal study of school belonging and academic motivation across high school. Child Development, 84(2), 678-692. https://doi.org/10.1111/j.14678624.2012.01862.x

Nur, İ., \& Aktaş Arnas, Y. (2019). Psychometric properties of the school liking and avoidance questionnaire in a turkish preschool sample. International Journal of Assessment Tools in Education, 6(4), 539-554. https://doi.org/10.21449/ijate.571664

Putwain, D. W., Schmitz, E. A., Wood, P., \& Pekrun, R. (2020). The role of achievement emotions in primary school mathematics: Control-value antecedents and achievement outcomes. British Journal of Educational Psychology. https://doi.org/10.1111/bjep.12367

Reddy, R., Rhodes, J. E., \& Mulhall, P. (2003). The influence of teacher support on student adjustment in the middle school years: A latent growth curve study. Development and Psychopathology 15, 119-138. https://doi.org/ 10.1017.S0954579403000075

Reeve, J. (2013). How students create motivationally supportive learning environments for themselves: The concept of agentic engagement. Journal of Educational Psychology, 105(3), 579-595. https://doi.org/10.1037/a0032690

Ribeiro, A. R.,Pereira, A. I., \& Pedro, M. (2019). Predictors of school affective engagement during elementary school: A systematic review. Análise Psicológica, 37(2), 193-207. https://doi.org/10.14417/ap.1547

Şahin-Sak, İ. T. (2018). Preschoolers' and first graders' reasons for liking or avoiding school. Education 313, 47, 889-905 . https://doi.org/10.1080/03004279.2018.1537300

Schneider, B. H., Manetti, M., Frattini, L., Rania, N., Santo, J. B., Coplan, R. J., \& Cwinn, E. (2014). Successful transition to elementary school and the implementation of facilitative practices specified in the Reggio-Emilia Philosophy. School Psychology International, 35(5), 447-462. https://doi.org/10.1177/0143034313511003

Skinner, E. A., \& Belmont, M. J. (1993). Motivation in the classroom: Reciprocal effects of teacher behavior and student engagement across the school year. Journal of Educational Psychology, 85(4), 571-581. https://doi.org/10.1037/0022-0663.85.4.571 
Smith, J. (2011). Measuring school engagement: A longitudinal evaluation of the school liking and avoidance questionnaire from kindergarten through sixth grade. Arizona State University.

Tomada, G., Schneider, B. H., de Domini, P., Greenman, P. S., \& Fonzi, A. (2005). Friendship as a predictor of adjustment following a transition to formal academic instruction and evaluation. International Journal of Behavioral Development, 29(4), 314-322. https://doi.org/10.1080/01650250544000099

Valeski, T. N., \& Stipek, D. J. (2001). Young Children's Feelings about School. Child Development, 72(4), 1198-1213. https://doi.org/10.1111/1467-8624.00342

Vassilopoulos, S. P., Brouzos, A., \& Koutsianou, A. (2018). Outcomes of a universal social and emotional learning (SEL) group for facilitating first-grade students' school adjustment. International Journal of School and $\quad$ Educational 223-236. https://doi.org/10.1080/21683603.2017.1327830

Veiga, F., Festas, I., Taveira, C., Galvão, D., Janeiro, I., Conboy, J., ... Nogueira, J. (2012). Envolvimento dos alunos na escola: conceito e relação com o desempenho académico - sua importância na formação de professores. Revista Portuguesa de Pedagogia, 46(2), 31-47.

Zhang, L., \& Eggum-Wilkens, N. D. (2018). Unsociability in Chinese adolescents: Cross-informant agreement and relations with social and school adjustment. Social Development, 27(3), 555-570. https://doi.org/10.1111/sode.12284

Zhang, X., Xuan, X., Chen, F., Zhang, C., Luo, Y., \& Wang, Y. (2016). The relationship among school safety, school liking, and students' self-esteem: Based on a multilevel mediation model. Journal of School Health, 86(3), 164-172. https://doi.org/10.1111/josh.12364

$\begin{array}{lr}\text { Historial do artigo } \\ \text { Recebido } & 01 / 2020 \\ \text { Aceite } & 10 / 2020 \\ \text { Publicado } & 12 / 2020\end{array}$

